\title{
CARACTERÍSTICAS QUÍMICAS DE UM LATOSSOLO ADUBADO COM URÉIA E CLORETO DE POTÁSSIO EM AMBIENTE PROTEGIDO
}

\author{
Maria Anita Gonçalves da Silva ${ }^{1 *}$; Antonio Enedi Boaretto²; Henriqueta Gimenes Fernandes²; \\ Rodrigo Boaretto²; Arlete Marchi Tavares de Melo ${ }^{3}$; Walkyria Bueno Scivittaro ${ }^{4}$ \\ ${ }_{2}^{1}$ Depto. de Agronomia - UEM, Av. Colombo 5790 - CEP: 87020-900 - Maringá, PR. \\ 2 Lab. de Fertilidade do Solo - USP/CENA, C.P. 96 - CEP: 13400-970 - Piracicaba, SP. \\ ${ }_{4}^{3}$ Seção de Horticultura - IAC, Fazenda Santa Elisa, C.P. 28 - CEP:13002-920 - Campinas, SP. \\ ${ }^{4}$ Embrapa Clima Temperado, Br 392, Km 78, C.P. 403 - CEP: 96001-970 - Pelotas, RS. \\ *Autor correspondente <magsilva@uem.br>
}

RESUMO: A produção de vegetais mais sadios e de boa qualidade e o fornecimento contínuo no mercado são fatores que têm determinado a adoção do sistema de cultivo protegido por um número maior de produtores. Porém, devido ao pouco conhecimento sobre o manejo do solo nessas condições, tem-se aplicado altas doses de fertilizantes, ocasionando problemas de salinidade e desequilíbrio nutricional. O objetivo desse trabalho foi avaliar os efeitos da aplicação da uréia e do cloreto de potássio nas características químicas de um LATOSSOLO VERMELHO Destrófico, principalmente nos índices de acidez e saturação em potássio. $O$ $\mathrm{N}$ e o $\mathrm{K}$ foram aplicados em cobertura, nas doses equivalentes de 13,3 e $39,9 \mathrm{~g} \mathrm{~m}^{-2}$ de $\mathrm{N}$ e 5,5 e $16,6 \mathrm{~g} \mathrm{~m}^{-2} \mathrm{de}$ $\mathrm{K}$, em esquema fatorial $(2 \times 2+1)$, com tratamento adicional, sem a aplicação dos nutrientes. Cultivou-se pimentão, cultivar Mayata, em condições de ambiente protegido, durante 34 semanas. Após o pegamento das mudas, foi aplicado $1 / 6$ dos nutrientes a cada dez dias. Ao final do ciclo, as formas de $\mathrm{N}$ nítrico e $\mathrm{N}$ amoniacal representaram melhor as doses do nutriente aplicados ao solo e não houve acidificação. As raízes tiveram significativo crescimento após a aplicação de $\mathrm{N}$, enquanto que o aumento da dose de $\mathrm{K}$, aplicado como $\mathrm{KCl}$, prejudicou o crescimento radicular, provavelmente associado à alta concentração de $\mathrm{K}$ no solo e possíveis efeitos salinos, correspondendo a mais de $5,0 \mathrm{mmol}_{\mathrm{c}} \mathrm{dm}^{-3}$ e mais que $5,3 \%$ de saturação do $\mathrm{K}$ no complexo de troca, na camada superficial do solo.

Palavras-chave: Capsicum annuum, adubação nitrogenada, adubação potássica, saturação de potássio

\section{CHEMICAL CHARACTERISTICS OF AN OXISOL AFTER UREA AND POTASSIUM CHLORIDE FERTILIZATION IN A PROTECTED ENVIRONMENT}

\begin{abstract}
The production of healthy, high quality vegetables and the development of technologies for continuous supply to the market are important factors determining the adaption of protected cultivation systems by an increasing number of growers. Due to the little knowledge on soil management practices under these conditions, high fertilizer rates are normally applied, causing salinity problems and nutricional unbalances. The objective of this paper was to evaluate the effects of urea and potassium chloride applications on the chemical characteristics of an Eutrorthox, mainly on its acidity and potassium saturation. The $\mathrm{N}$ was applied in rates of 13.3 and $39.9 \mathrm{~g} \mathrm{~m}^{-2}$ and the $\mathrm{K}$ in rates 5.5 and $16.6 \mathrm{~g} \mathrm{~m}^{-2}$, in a fatorial $(2 \times 2+1)$ design, including a control. Pepper plants (Capsicum annuum, Mayata cultivar) were grown under protected condition, during 34 weeks. After seedling rooting, the nutrients were applied at the rate of one sixth of the total at ten day intervals, up to 60 days. It was observed that urea did not acidify the soil probabily due to its high buffering capacity. The nitric and ammoniacal forms of $\mathrm{N}$ were directly effeted by the $\mathrm{N}$ rates applied in relation to the soil. In the treatment with $\mathrm{N}$, the roots showed better development compared to the control. However, the highest $\mathrm{K}$ rate, applied as $\mathrm{KCl}$, decreased root growth, probably due to the high soil $\mathrm{K}$ concentration and possible salinity effects, resulting in more than $5.0 \mathrm{mmol}_{\mathrm{c}} \mathrm{dm}^{-3}$ of $\mathrm{K}$ and more than $5.3 \%$ of $\mathrm{K}$ saturation in the exchange complex.

Key words: Capsicum annuum, nitrogen, potassium, potassium saturation
\end{abstract}

\section{INTRODUÇÃO}

Em decorrência da sazonalidade dos produtos hortícolas, os agricultores têm sido levados a melhorar os sistemas de cultivo, de modo a obter colheitas em períodos de baixa oferta destes produtos no mercado interno. O cultivo em ambiente protegido possibilita produção contínua e certa, abastecendo o mercado o ano todo. Nesse sistema, tem-se conseguido colheitas com alto rendimento e boa qualidade dos produtos, em épocas do ano em que as condições normalmente são desfavoráveis no campo. Desse modo, é possível abastecer e alcançar mercado nas estações dos meses relativos ao inverno e à primavera. Várias hortaliças 
estão sendo produzidas nesse ambiente protegido, em túneis de plástico, entre elas o pimentão. Nesse ambiente, o comportamento da planta e sua necessidade nutricional são diferentes daquelas que ocorrem em condições de campo. Apesar disso, as mesmas doses de fertilizantes são indicadas para os dois sistemas de cultivo, enfatizando a necessidade de pesquisas sobre este assunto, que servirão de base para que os fertilizantes sejam usados pelos produtores de forma mais racional e econômica. Nesse sentido, em condições de cultivo protegido, Qawasmi et al. (1999) encontraram efeito favorável à aplicação de $\mathrm{N}$ no crescimento e produção de pimentão, durante 180 dias de cultivo. Nas mesmas condições de cultivo, Moura et al. (1999) referem-se à importância do $\mathrm{P}$ para o pimentão, enquanto Valenzuela \& Romero (1996) avaliaram o efeito favorável do $\mathrm{N}$ e $\mathrm{K}$, aplicados através de fertirrigação, no redimento dos frutos.

O uso de fertilizantes também deve ser criterioso, principalmente em relação ao potássio, aplicado como $\mathrm{KCl}$, porque pode resultar em alta concentração de sais em solução, além de elevar a saturação do K no complexo coloidal do solo, causando desequilíbrio iônico.

Os objetivos do trabalho foram avaliar o efeito da uréia sobre os teores de $\mathrm{N}$ total, $\mathrm{N}$ mineral e sobre $\mathrm{o}$ crescimento das raízes do cloreto de potássio na concentração e saturação de $\mathrm{K}$ do solo, bem como avaliar o efeito salino do $\mathrm{KCl}$ no solo, após 34 semanas de cultivo do pimentão em ambiente protegido.

\section{MATERIAL E MÉTODOS}

O pimentão, cultivar Mayata, foi cultivado durante 34 semanas em um Latossolo Vermelho Distrófico (Embrapa, 1999), em ambiente protegido, tipo túnel plástico, com telas nas laterais e área de $210 \mathrm{~m}^{2}$, no Centro Experimental IAC-Campinas, SP.

No início do experimento, antes da adubação, o solo, com $590 \mathrm{~g} \mathrm{~kg}^{-1}$ de argila e $333 \mathrm{~g} \mathrm{~kg}^{-1}$ de areia, apresentou na camada de $0-20 \mathrm{~cm}$ de profundidade, as seguintes características químicas: $\mathrm{pH}\left(\mathrm{CaCl}_{2}\right)=5,7$; matéria orgânica $=24,0 \mathrm{~g} \mathrm{dm}^{-3} ; \mathrm{P}$ (resina) $=199,0 \mathrm{mg}$ $\mathrm{dm}^{-3} ; \mathrm{S}^{-S} \mathrm{O}_{4}=50,7 \mathrm{mg} \mathrm{dm}^{-3} ; \mathrm{N}$ total $=1,8 \mathrm{~g} \mathrm{~kg}^{-1} ; \mathrm{K}$ (resina) $=3,2 \mathrm{mmol}_{\mathrm{c}} \mathrm{dm}^{-3} ;$ Ca trocável $=52,0 \mathrm{mmol}_{\mathrm{c}} \mathrm{dm}^{-3} ; \mathrm{Mg}$ trocável $=19,0 \mathrm{mmol}_{\mathrm{c}} \mathrm{dm}^{-3} ; \mathrm{H}^{+}+\mathrm{Al}^{3+}=22,0 \mathrm{mmol}_{\mathrm{c}} \mathrm{dm}^{-3}$; soma de bases $=74,2 \mathrm{mmol}_{\mathrm{c}} \mathrm{dm}^{-3} ;$ CTC $=96,2 \mathrm{mmol}_{\mathrm{c}}$ $\mathrm{dm}^{-3}$; saturação de bases $=77 \%$ e saturação de $\mathrm{K}=$ $3,3 \%$. Para a camada de 20 a $40 \mathrm{~cm}$, os resultados foram: $\mathrm{pH}\left(\mathrm{CaCl}_{2}\right)=4,8$; matéria orgânica $=24,0 \mathrm{~g} \mathrm{dm}^{-3}$; $\mathrm{P}($ resina $)=39,0 \mathrm{mg} \mathrm{dm}^{-3} ; \mathrm{S}^{-\mathrm{SO}_{4}}=134,3 \mathrm{mg} \mathrm{dm}^{-3} ; \mathrm{N}$ total $=1,7 \mathrm{~g} \mathrm{~kg}^{-1} ; \mathrm{K}$ (resina) $=3,5 \mathrm{mmol}_{\mathrm{dm}}^{-3} ;$ Ca trocável $=$ $27,0 \mathrm{mmol}_{\mathrm{c}} \mathrm{dm}^{-3} ; \mathrm{Mg}$ trocável $=14,0 \mathrm{mmol}_{\mathrm{c}} \mathrm{dm}^{-3} ; \mathrm{H}^{+}+\mathrm{Al}^{3+}$ $=34,0 \mathrm{mmol}_{\mathrm{c}} \mathrm{dm}^{-3}$; soma de bases $=45,0 \mathrm{mmol}_{\mathrm{c}} \mathrm{dm}^{-3}$; CTC $=79,0 \mathrm{mmol}_{\mathrm{c}} \mathrm{dm}^{-3}$; saturação de bases $=57 \%$ e a saturação de $K=4,4 \%$. As análises foram feitas segundo metodologia descrita por Raij et al. (1987). A acidez potencial, foi determinada através de extração com acetato de Ca centimolar, tamponado a pH 7,0. O fósforo foi extraído pelo método da resina saturada com bicarbonato de sódio $0,5 \mathrm{M}$ e determinado por colorimetria. A mesma forma de extração foi usada para o potássio, sendo determinado por fotometria de chama.

Foram aplicados em área total, adubo orgânico (esterco de curral), em quantidade relativa a $20 \mathrm{t} \mathrm{ha}^{-1}$ de peso úmido (Nagai, 1995), contendo 5,0 e $6,0 \mathrm{~g} \mathrm{~kg}^{-1}$ de $\mathrm{N}$ e K, respectivamente e calcário dolomítico, objetivando elevar a saturação por bases a $80 \%$ e o teor de Mg para atingir $9,0 \mathrm{mmol}_{\mathrm{c}} \mathrm{dm}^{-3}$. Nos canteiros foram aplicados 100 $\mathrm{mg} \mathrm{m}^{-2}$ de B (bórax) e $240 \mathrm{mg} \mathrm{m}^{-2}$ de $\mathrm{Zn}$ (sulfato de zinco), conforme recomendação de Boaretto (1986) e Raij et al. (1996).

Os tratamentos em esquema fatorial, com tratamento adicional $(2 \times 2+1)$ corresponderam a duas doses de nitrogênio $\left(13,3\right.$ e $\left.39,9 \mathrm{~g} \mathrm{~m}^{-2}\right)$, aplicado na forma de uréia e duas doses de $\mathrm{K}\left(5,5\right.$ e 16,6 $\left.\mathrm{g} \mathrm{m}^{-2}\right)$, aplicado como $\mathrm{KCl}$ e um tratamento, sem a aplicação dos nutrientes (tratamento controle), aplicados em 6 vezes, entre o período de 13/11/96 a 24/01/97, iniciando-se 13 dias após o transplante das mudas.

Os adubos foram aplicados nas linhas abaixo dos gotejadores, sendo incorporado ao solo através da água irrigada. $O$ volume de água mínimo para o pimentão correspondeu a um potencial matricial de 11 $\mathrm{kPa}$ e foi calculado em função da curva de retenção de água pelo solo (Wosten \& van Genuchten, 1988). O delineamento experimental adotado foi o de blocos ao acaso, com 4 repetições. Cada unidade experimental foi constituída de 11 plantas, sendo consideradas úteis as 7 plantas centrais. A área da parcela foi de 2,75 $\mathrm{m}^{2}$, considerando duas linhas de plantas, espaçadas de 0,50 $\mathrm{m}$ na linha e 1,0 m na entrelinha .

As amostras de solo foram coletadas nas profundidades de $0-20$ e $20-40 \mathrm{~cm}$, no centro e nas duas laterais da linha de gotejo. De cada parcela foram retiradas 5 amostras simples para formar uma amostra composta. O solo foi seco ao ar e moído. Foram determinadas a acidez ativa e potencial, conteúdo de matéria orgânica e potássio trocável, conforme metodologia proposta por Raij et al. (1987). O N total, N amoniacal e o $\mathrm{N}$ nítrico foram analisados segundo Bremner \& Mulvaney (1982). o N total foi convertido em sulfato de amônio através de oxidação com uma mistura ácida e determinado por destilação da amônia. As formas inorgânicas foram extraídas com cloreto de potássio 2,0 mol L $\mathrm{L}^{-1}$ e determinados por destilação e titulação da amônia com ácido sulfúrico.

A condutividade elétrica dos solos foi determinada segundo metodologia de Camargo et al. (1986).

Para a coleta das raízes adotou-se a metodologia de Bohn (1979), com algumas modificações introduzidas pelo Centro de Ecofisiologia e Biofísica do IAC. Utilizou-se trado específico, com volume de 407 $\mathrm{cm}^{3}$. O peso do solo úmido correspondeu em média a 
$500 \mathrm{~g}$, com umidade entre 25 a $30 \%$, dependendo do dia de coleta. As raízes foram coletadas logo abaixo da linha de gotejo, nas profundidades de $0-10,10-20$ e $20-40 \mathrm{~cm}$. Foram feitas 2 amostragens por planta, utilizando-se 3 plantas por parcela. $O$ trado foi colocado a uma distância de $10 \mathrm{~cm}$ do caule da planta. A separação das raízes do solo foi feita por meio de dispersão em água e peneiramento segundo metodologia de Fujimura et al. (1994). As raízes foram lavadas e colocadas para secar à sombra sobre papel toalha. Após a secagem, fez-se a separação das impurezas, tais como raízes de ervas daninhas e material orgânico. A seguir, o material foi seco em estufa a $65^{\circ} \mathrm{C}$ por $48 \mathrm{hs}$ determinando-se, o peso da matéria seca.

Os tratamentos amostrados, para as análises químicas e de raízes, foram aqueles onde o menor nível de $\mathrm{N}$ estava combinado com o menor e com o maior nível de $\mathrm{K}$, bem como a combinação entre as doses máximas entre os nutrientes. O mesmo procedimento foi adotado na escolha dos tratamentos com $\mathrm{K}$, além da amostra controle, sem aplicação de N e K. O objetivo da escolha desses tratamentos, foi verificar a limitação no crescimento das raízes, por um possível efeito de salinidade do $\mathrm{KCl}$.

Utilizou-se o teste de Dunnett bilateral ao nível de $5 \%$, para comparar a testemunha com os demais tratamentos; para comparar os teores de amônio e nitrato foi utilizado o teste de Tukey a $5 \%$ e para comparar as doses de $\mathrm{K}$ foi usado o teste $\mathrm{F}$.

\section{RESULTADOS E DISCUSSÃO}

A aplicação de adubos no solo pode causar efeitos desejáveis e indesejáveis em suas propriedades químicas. A alteração desejável é aumentar o teor no solo do nutriente aplicado na adubação, quando este limita o crescimento vegetal, possibilitando que a cultura manifeste todo o seu potencial genético de produtividade.

Para a uréia, o principal efeito indesejável é o possível aumento da acidez do solo (Malavolta, 1981; Maclaren \& Cameron, 1996). No presente experimento, o efeito da aplicação da uréia na acidez do solo foi mínimo, pois o pH que era em média, antes do início do cultivo de pimentão, de 5,7 e 4,8 nas camadas de 0 20 e $0-40 \mathrm{~cm}$, respectivamente, passou para 5,6 e 5,2 nas mesmas camadas, ao final do ciclo (TABELA 1). Da mesma forma, não houve diferença significativa entre tratamentos com $\mathrm{N}$ e a testemunha, sem o nutriente, em relação à acidez potencial do solo. As médias de acidez potencial entre os tratamentos foram de 27,7 e 33,9 $\mathrm{mmol}_{\mathrm{c}} \mathrm{dm}^{-3}$ e na testemunha a acidez potencial foi de 26,8 e $35,1 \mathrm{mmol}_{\mathrm{c}} \mathrm{dm}^{-3}$, respectivamente para 0-20 e 20$40 \mathrm{~cm}$ de profundidade de amostragem. Deve-se considerar que o solo usado tem textura argilosa até a profundidade de $40 \mathrm{~cm}$, o que deve ter evitado que alterações mais drásticas ocorressem. Além disso o médio conteúdo de matéria orgânica também contribuiu para tamponar o meio, mantendo o pH estável. O teor médio de matéria orgânica entre os tratamentos esteve entre 23,2 e $22,1 \mathrm{~g} \mathrm{dm}^{-3}$ a 20 e $40 \mathrm{~cm}$ no solo, sem diferenciar-se da testemunha.

Observaram-se valores relativamente altos de condutividade elétrica ( $C E$ ), nas duas profundidades amostradas, nos tratamentos com adição de $16,6 \mathrm{~g} \mathrm{~m}^{-2}$ de $\mathrm{K}$, propiciando um ambiente mais salino em torno das raízes, comprometendo o seu crescimento e a absorção de água e nutrientes. Papadoulos \& Khosla (1993) verificaram que houve diminuição da CE de 3800 para $1100 \mathrm{mS} \mathrm{cm}^{-1} \mathrm{com}$ a diminuição do nível de $\mathrm{K}$ em solução nutritiva, não prejudicando o crescimento das raízes e rendimento de frutos de tomate, após 28 semanas de cultivo em ambiente protegido.

A aplicação de $\mathrm{N}$ resultou em aumento significativo do teor de $\mathrm{N}$ total apenas nas camadas de 20 a $40 \mathrm{~cm}$, comparativamente à testemunha, sem diferença entre as doses aplicados (TABELA 2). Os valores médios encontrados em ambas as profundidades foram de 1,38 e $1,18 \mathrm{~g} \mathrm{~kg}^{-1}$, respectivamente, menores que os teores encontrados por Gianello et al. (2000).

Silva et al. (1999) determinaram teores de $\mathrm{N}$ total entre 0,9 e $3,3 \mathrm{~g} \mathrm{~kg}^{-1}$ em solos brasileiros. Segundo Malavolta et al. (1997) solos de cerrado apresentam teores médios de $\mathrm{N}$ total de 0,8 a $1,4 \mathrm{~g} \mathrm{dm}^{-3}$.

Se considerado o teor de N inorgânico, (TABELA 2) verificou-se, que tanto o teor de amônio quanto o teor de nitrato foram maiores com as doses de uréia, embora os teores de nitrato foram mais baixos que os teores referidos por Vasconcellos et al. (1998). Porém concentração do $\mathrm{N}$ amoniacal no solo com o pimentão, foi dobro, comparativamente aos teores encontrados pelos mesmos autores. Outros autores como Locascio \& Alliggod (1992), Jaworski et al. (1978), Batal \& Smittle (1981), Locascio et al. (1981), Hochmuth et al. (1987) e Hochmuth et al. (1994) encontraram variações nos teores de nitrato e amônio do solo, motivadas pela adubação nitrogenada, que superam os teores encontradas nesse trabalho. De acordo com Silva et al. (1999) solos brasileiros tiveram teores de $\mathrm{N}$ mineral $\left(\mathrm{NH}_{4}{ }^{+}+\mathrm{NO}_{3}{ }^{-}\right)$de $10,3 \mathrm{mg} \mathrm{dm}^{-3}$.

O N amoniacal, comparativamente ao nítrico, concentrou-se mais na camada de 0 a $20 \mathrm{~cm}$, provavelmente mais adsorvido à matéria orgânica e às argilas. A presença de amônio e nitrato de 20 a $40 \mathrm{~cm}$ pode ter sido decorrente da movimentação dos íons no solo, quando em quantidades mais altas. Goldberg et al. (1971) verificaram que o nitrato movimentou-se no solo junto com a água aplicada pelos gotejadores, assim como Kafkafi \& Bar-Yosef (1980) determinaram alta concentração de nitrato (100 $\mathrm{mg} \mathrm{g}^{-1}$ de solo) a profundidade de 20 a $40 \mathrm{~cm}$ abaixo do gotejador.

A maior massa das raízes esteve presente até $20 \mathrm{~cm}$ (TABELA 3), com a aplicação de nitrogênio. O sistema radicular desenvolveu-se menos, quando o $\mathrm{K}$ foi aplicado na dose de $16,6 \mathrm{~g} \mathrm{~m}^{-2}$, possivelmente devido 
ao efeito salino do KCl. Persaud et al. (1977) relacionam a presença de raízes mais finas e subdivididas, em tomate irrigado, à altas concentrações de sais potássicos no solo.

Ao final do ciclo de cultivo do pimentão, o K trocável foi significativamente maior conforme sua aplicação ao solo, nas duas profundidades amostradas (Figura 1), confirmando a sua permanência no solo, principalmente adsorvido aos colóides orgânicos e inorgânicos.

Teores menores de $\mathrm{K}$ trocável foram encontrados por Hortenstine (1962) e Fiskell et al. (1978). Estes referem-se a $2,15 \mathrm{mmol}_{\mathrm{c}} \mathrm{dm}^{-3}$ de $\mathrm{K}$ no solo, extraído por duplo ácido, após 17 semanas de cultivo. Hochmuth et al. (1994) encontraram teores de $\mathrm{K}$ até $0,59 \mathrm{mmol}_{\mathrm{c}} \mathrm{dm}^{-3}$ na linha de plantio e até $3,28 \mathrm{mmol}_{\mathrm{c}} \mathrm{dm}^{-3}$ na entre linha.

$\mathrm{O}$ aumento do $\mathrm{K}$ no solo refletiu-se na saturação do nutriente no complexo de troca (Figura 2). Ao final do experimento, a saturação de $\mathrm{K}$ na camada de 0-20 $\mathrm{cm}$, que era de $2,2 \%$ na testemunha sem $\mathrm{K}$, subiu para $5,6 \%$, quando as maiores doses do nutriente foram aplicadas. Entre 20 e $40 \mathrm{~cm}$ aumentou para 6,8\%, demonstrando a movimentação do $\mathrm{K}$ no solo, mesmo com médios teores de matéria orgânica no solo e textura mais argilosa. $\mathrm{O}$ aumento da saturação pelo $\mathrm{K}$ aplicado como $\mathrm{KCl}$, pode ter sido o responsável pelo baixo crescimento das raízes, verificado na TABELA 3. Destes resultados, pode-se deduzir que teores de $\mathrm{K}$ de 5,0 a 7,0 $\mathrm{mmol}_{\mathrm{c}} \mathrm{dm}^{-3}$ de solo (Figura 1), que nesse trabalho corresponderam a uma saturação de $\mathrm{K}$ de 5,3 e 6,6\% (Figura 2), não devem ser ultrapassados, quando se deseja cultivar pimentão no solo sob condições protegidas, pois a lixiviação é menor que em condições normais, ocorrendo acúmulo de sais. A salinização do solo vem acontecendo com freqüência sob cultivo em ambiente protegido (Tadesse et al., 1999; Richter et al., 1999).

De forma contrária, Santiago \& Goyal (1985) relatam que a concentração de $\mathrm{K}$ diminuiu entre 15 a 45 $\mathrm{cm}$ no solo, após cultivo de pimentão irrigado, devido à maior presença de raízes nessa área e a consequente maior absorção do nutriente.

TABELA 1 - Características químicas do solo, em duas profundidades, ao final do cultivo do pimentão (34 semanas). Média de 4 repetições.

\begin{tabular}{|c|c|c|c|c|c|c|c|c|c|}
\hline \multirow[t]{2}{*}{$\mathrm{N}$} & \multirow[t]{2}{*}{$\mathrm{K}$} & \multicolumn{2}{|c|}{$\mathrm{pH}$} & \multicolumn{2}{|c|}{ Acidez Potencial } & \multicolumn{2}{|c|}{ Matéria Orgânica } & \multicolumn{2}{|c|}{ C E } \\
\hline & & $0-20$ & $20-40$ & $0-20$ & $20-40$ & $0-20$ & $20-40$ & $0-20$ & $20-40$ \\
\hline \multicolumn{2}{|c|}{----- $\mathrm{g} \mathrm{m}^{-2}$} & & & \multicolumn{2}{|c|}{---- $\mathrm{mmol}_{\mathrm{c}} \mathrm{dm}^{-3}$} & \multicolumn{2}{|c|}{---- $\mathrm{g} \mathrm{dm}^{3}$} & \multicolumn{2}{|c|}{----- $\mu \mathrm{S} \mathrm{cm}^{-1}$} \\
\hline 0,0 & 0,0 & 5,7 & 5,2 & 26,8 & 35,1 & 23,0 & 19,7 & $496,5 \mathrm{a}$ & $603,3 \mathrm{a}$ \\
\hline 13,3 & 5,5 & $5,5 \mathrm{~ns}^{(2)}$ & $5,2 \mathrm{~ns}$ & $29,4 \mathrm{~ns}$ & $34,4 \mathrm{~ns}$ & $24,4 \mathrm{~ns}$ & $20,8 \mathrm{~ns}$ & $517,0 \mathrm{a}$ & $759,0 \mathrm{a}$ \\
\hline 13,3 & 16,6 & $5,7 \mathrm{~ns}$ & $5,5 \mathrm{~ns}$ & $26,3 \mathrm{~ns}$ & $30,6 \mathrm{~ns}$ & $23,3 \mathrm{~ns}$ & $22,3 \mathrm{~ns}$ & $972,0 \mathrm{~b}$ & $1224,5 b$ \\
\hline 39,9 & 5,5 & $5,5 \mathrm{~ns}$ & $5,2 \mathrm{~ns}$ & $28,1 \mathrm{~ns}$ & $36,0 \mathrm{~ns}$ & $22,6 \mathrm{~ns}$ & $20,1 \mathrm{~ns}$ & $712,5 \mathrm{a}$ & $622,5 \mathrm{a}$ \\
\hline 39,9 & 16,6 & $5,6 \mathrm{~ns}$ & $5,2 \mathrm{~ns}$ & $27,8 \mathrm{~ns}$ & $33,6 \mathrm{~ns}$ & $23,0 \mathrm{~ns}$ & $21,5 \mathrm{~ns}$ & $873,5 b$ & $1138,5 b$ \\
\hline Média & & 5,6 & 5,2 & 27,7 & 33,9 & 23,2 & 22,1 & 714,3 & 869,6 \\
\hline CV (\%) & & 4 & 5 & 14 & 14 & 6 & 13 & 13 & 8 \\
\hline
\end{tabular}

(1)Diferenças significativas ou ${ }^{(2)}$ diferenças não significativas a $5 \%$ pelo teste de Dunnett, entre as médias do tratamento em relação à testemunha.

TABELA 2 - Concentração de amônio e nitrato, em duas profundidades do solo, após 34 semanas de cultivo com pimentão, em função de doses de nitrogênio aplicado ao solo. Média de 4 repetições.

\begin{tabular}{|c|c|c|c|c|c|c|c|}
\hline \multirow[t]{2}{*}{$\mathrm{N}$} & \multirow[t]{2}{*}{ K } & \multicolumn{2}{|c|}{$\mathrm{N}$ total } & \multicolumn{2}{|c|}{$\mathrm{N}-\mathrm{NH}_{4}^{+}$} & \multicolumn{2}{|c|}{$\mathrm{N}^{-\mathrm{NO}_{3}^{-}}$} \\
\hline & & $0-20$ & $20-40$ & $0-20$ & $20-40$ & $0-20$ & $20-40$ \\
\hline \multicolumn{2}{|c|}{ 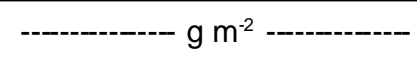 } & \multicolumn{2}{|c|}{ - } & \multicolumn{4}{|c|}{ - } \\
\hline 0 & 0 & $1,36 \mathrm{a}$ & $1,10 \mathrm{a}$ & $2,01 a^{(1)}$ & $2,39 \mathrm{a}$ & $0,61 \mathrm{a}$ & $1,19 \mathrm{a}$ \\
\hline 13,3 & 5,5 & $1,36 \mathrm{a}$ & $1,21 b$ & $6,09 \mathrm{~b}$ & $3,01 \mathrm{a}$ & $1,81 \mathrm{~b}$ & $1,52 \mathrm{a}$ \\
\hline 39,9 & 16,6 & $1,41 \mathrm{a}$ & $1,25 b$ & $5,35 \mathrm{~b}$ & $4,22 \mathrm{~b}$ & $2,58 \mathrm{c}$ & $4,85 \mathrm{~b}$ \\
\hline Médias & & 1,38 & 1,18 & 4,48 & 3,21 & 1,67 & 2,52 \\
\hline CV (\%) & & 5 & 5 & 14 & 12 & 20 & 12 \\
\hline
\end{tabular}

(1)Médias seguidas da mesma letra na coluna, não diferem a 5\% pelo teste de Tukey. 
TABELA 3 - Peso da matéria seca de raízes do pimentão em função de doses de $\mathrm{N}$ e K aplicadas ao solo. Média de 4 repetições.

\begin{tabular}{|c|c|c|c|c|}
\hline \multirow[t]{2}{*}{$\mathrm{N}$} & \multirow[t]{2}{*}{$\mathrm{K}$} & \multicolumn{3}{|c|}{ Profundidade da amostra do solo $(\mathrm{cm})$} \\
\hline & & $0-10$ & $10-20$ & $20-40$ \\
\hline \multicolumn{2}{|c|}{$----g^{-2}$} & ------------- & $--g$ & ----------- \\
\hline 0,0 & 0,0 & 0,37 & 0,22 & 0,04 \\
\hline 13,3 & 5,5 & $0,59 * *(1)$ & 0,42 ** & 0,12 ** \\
\hline 13,3 & 16,6 & $0,45 \mathrm{~ns}^{(2)}$ & $0,26 \mathrm{~ns}$ & $0,05 \mathrm{~ns}$ \\
\hline 39,9 & 5,5 & $0,54 *(1)$ & 0,40 ** & 0,11 * \\
\hline 39,9 & 16,6 & $0,49 \mathrm{~ns}$ & $0,27 \mathrm{~ns}$ & 0,09 * \\
\hline Média & & 0,49 & 0,31 & 0,08 \\
\hline CV (\%) & & 17 & 17 & 31 \\
\hline
\end{tabular}

(1)Diferenças significativas a $5 \%\left(^{*}\right)$ e $1 \%\left(^{(*}\right)$ ou ${ }^{(2)}$ diferenças não significativas, pelo teste de Dunnett, entre as médias dos tratamentos e a testemunha.

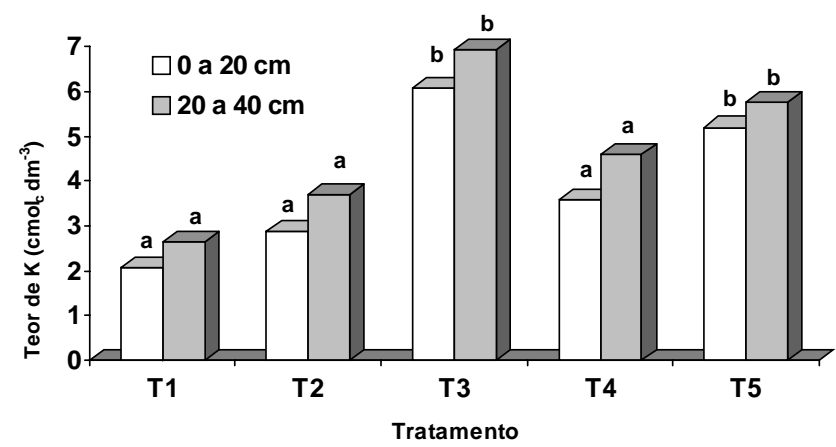

Figura 1 - Concentração de potássio no solo, às profundidades de 0 a $20 \mathrm{~cm}$, e 20 a $40 \mathrm{~cm}$, após 34 semanas de cultivo do pimentão. T1- testemunha; T2- $13,3 \mathrm{~g} \mathrm{~m}^{-2}$ de $\mathrm{N}$ e $5,5 \mathrm{~g} \mathrm{~m}^{-2}$ de K; T3-13,3 $\mathrm{g} \mathrm{m}^{-2}$ de $\mathrm{N}$ e $16,6 \mathrm{~g} \mathrm{~m}^{-2}$ de K; $\mathrm{T} 4-39,9 \mathrm{~g} \mathrm{~m}^{-2}$ de N e $5,5 \mathrm{gm}^{-2}$ de K; T5 - 39,9 $\mathrm{g} \mathrm{m}^{-2}$ de $\mathrm{N}$ e $16,6 \mathrm{~g} \mathrm{~m}^{-2}$ de K. Média de 4 repetições. Em cada profundidade, valores com mesma letra não diferem significativamente pelo teste Tukey a $5 \%$.

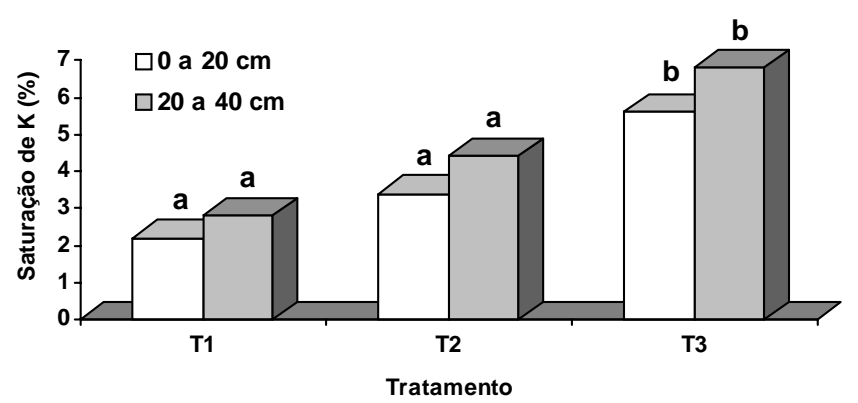

Figura 2 - Saturação de potássio no solo, às profundidades de 20 e $40 \mathrm{~cm}$, após 34 semanas de cultivo do Pimentão. $\mathrm{T} 1$ - testemunha sem $\mathrm{K}$; T2-5,5 $\mathrm{g} \mathrm{m}^{-2}$ de $\mathrm{K}$; T3 $16,6 \mathrm{~g} \mathrm{~m}^{-2}$ de K. Média de 4 repetições. Em cada profundidade, valores com mesma letra não diferem significativamente pelo teste Tukey a $5 \%$.

\section{CONCLUSÕES}

Ao final do cultivo do pimentão, após 34 semanas, os teores de $\mathrm{N}$ nítrico e $\mathrm{N}$ amoniacal representaram melhor o nutriente no solo, aplicado através da uréia. $\mathrm{O}$ $\mathrm{N}$ favoreceu o crescimento das raízes.

$\mathrm{OK}$ aplicado como $\mathrm{KCl}$, na dose de $16,6 \mathrm{~g} \mathrm{~K} \mathrm{~m}^{-2}$ prejudicou o crescimento das raízes, provavelmente associado à sua alta saturação no solo e possíveis efeitos salinos, correspondendo a teores entre 5,0 e 7,0 $\mathrm{mmol}_{\mathrm{c}} \mathrm{dm}^{-3}$ e de saturação do $\mathrm{K}$ no complexo de troca entre 5,3 e $6,6 \%$.

\section{AGRADECIMENTOS}

Ao Engenheiro Agrônomo Flávio Pagani de Castro e ao Sr. Assis Lopes dos Santos pelo apoio nos trabalhos de campo e ao Prof. Dr. Takashi Muraoka pela amizade e ensinamentos que muito contribuíram para a realização deste trabalho. À FAPESP - S.P., pelo financiamento desta pesquisa

\section{REFERÊNCIAS BIBLIOGRÁFICAS}

BATAL, K.M.; SMITTLE, D.A. Response of bell pepper to irrigation, nitrogen and plant population. Journal of the American Society for Horticultural Science, v.106, p.259262, 1981.

BOARETTO, A.E. Análise química de terra e recomendação de calagem e adubação para as principais culturas do Estado de São Paulo. Botucatu: Fundação de Estudos Agrícolas e Florestais,1986.50p. (Boletim Didático, 2).

$\mathrm{BOHN}, \mathrm{W}$. Methods of studying root systems. Berlin: SpringerVerlag, 1979. 189p.

BREMNER, J.M.; MULVANEY, C.S. Nitrogen total. In: PAGE, A.L.; MILLER, R.H.; KEENEY, D.R. (Ed). Methods of soil analisys: chemical and microbiological properties. 2 ed. Madison: ASA, v.2, cap.31, p.595-624, 1982. (Agronomy Series of Monographs, 9).

CAMARGO, O.A. de; MONIZ, A.C.; JORGE, J.A.; VALADARES, J.M.A.S. Métodos de análise química, mineralógica e física de solos do Instituto Agronômico de Campinas. Campinas: IAC, SDTC, 1986. 93p. (Boletim Técnico, 106).

EMBRAPA. Centro Nacional de Pesquisa do Solo. Sistema brasileiro de classificação de solos. Rio de Janeiro, 1999. 412p.

FISKELL, J.G.A.; LOCASCIO, S.J.; SINGHOLKA, S.; MARTIN, F.G. Effects of fertilization $\mathrm{N}$ sources, rates and placement on soil test values for bedded peppers with and without mulch. Soil and Crop Science Society of Florida Annual Proceedings, v.37, p.183-188, 1978.

FUJIMURA, M.; KURACHI, S.A.H.; ARRUDA, F.B.; PIRES, R.C. de $M$. A técnica de estudo de raízes pelo método do trado. Campinas: IAC, 1994. 10p. (Boletim Técnico, 153).

GIANELLO, C.; CAMARGO, F.A.O.; REICHMANN, E.; TEDESCO, M.J. Avaliação da disponibilidade do nitrogênio do solo estimada por métodos químicos. Revista Brasileira de Ciência do Solo, v.24, p.93-101, 2000.

GOLDBERG, D.; GORNAT, B.; BAR-YOSEF, B. The distribution of roots, water and minerals as a result of trickle irrigation. Journal of the American Society for Horticultural Science, v.96, p.645-648, 1971. 
HOCHMUTH, G.J.; SHULER, K.D.; MITCHELL, R.L.; GILREATH, P.R. Nitrogen crop nutrient requirement demonstrations for mulched pepper in Florida. Proceedings of the Florida State for Horticultural Society, v.100, p.205-209, 1987.

HOCHMUTH, G.J.; SHULER, K.; HANLON, E.; ROE, N. Pepper response to fertilization with soluble and controlled release potassium fertilizers. Proceedings of the Florida State of Horticultural Society, v.107, p.132-139, 1994.

HORTENSTINE, C.C. The response of sweet peppers in everglades organic soils to $\mathrm{P}$ and $\mathrm{K}$ fertilization. Proceedings of the Florida State for Horticultural Society, v.75, p.146$152,1962$.

JAWORSKI, C.A.; KAYS, S.J.; SMITLE, D.A . Effects of nitrogen and potassium fertilization in trickle irrigation on yield of pepper and polebean. Hortscience, v.13, p.477-478, 1978.

KAFKAFI, V.; BAR-YOSEF, B. Trickle irrigation and fertilization of tomatoes in hight calcareous soils. Agronomy Journal, v.72, p.873-897, 1980.

LOCASCIO, S.J.; ALLIGOOD, M.R. Nitrogen and potassium source and N-rate for drip-irrigated pepper. Proceedings of the Florida State for Horticulture Society, v.105, p.323-325, 1992.

LOCASCIO, S.J.; FISKELL, J.G.A; MARTIN, F.G. Responses of bell pepper to nitrogen sources. Journal of the American Society for Horticultural Science, v.106, p.628-632, 1981.

MACLAREN, R.G.; CAMERON, K.C. Soil, plant and fertilizer nitrogen. In: McLAREN, R.G. (Ed.) Soil science: Sustainable production and environmental protection. 2.ed. New York: Oxford University Press, 1996. p.192-207.

MALAVOLTA, E. Manual de química agrícola: adubos e adubação. 3.ed. São Paulo: Agronômica Ceres, 1981. 594 p.

MALAVOLTA, E.; VITTI, G.C.; OLIVEIRA, S.A. de. Avaliação do estado nutricional de plantas: princípios e aplicações. 2.ed. Piracicaba: Potafos, 1997. 319p.

MOURA, W.D.; CASALI, V.W.D.; CRUZ, C.D.; DE LIMA, P.C. Genetic divergence of phosphorus nutrition efficiency in sweet pepper lines. Pesquisa Agropecuária Brasileira v.34, p.217224, 1999.

NAGAI, H. Pimentão. In: FAHL, J.I.; CAMARGO, M.B.P. de; PIZZINATTO, M.A.; FURLANI, A.M.C.; BETTI, J.A.; MELLO, A.M.T. de; MARIA, I.C. de. (Ed.) Instruções agrícolas para - Estado de São Paulo. 6.ed. Campinas: Instituto Agronômico, 1995. p.335-337. (Boletim 200).

PAPADOULOS, A.P.; KHOSLA, S. Effect of nitrogen and potassium in tomatoes grown in nutritive solution. Canadian Journal of Plant and Science, v.73, p.289-296, 1993.

PERSAUD, N.; LOCASCIO, S.J.; GERALDSON, C.M. Influence of fertilizer rate and placement and irrigation method of plant nutrient status, soil soluble salt and root distribuition of mulched tomatoes. Soil and Crop Science Society of Florida, v.36, p.121-125, 1977.
QAWASMI, W.; MOHAMMAD M.J.; NAJIM, H.; QUBURSI, R. Response of bell pepper grown inside plastic house to nitrogen fertilization. Communications in Soil Science and Plant Analysis, v.30, p.2499-2509, 1999.

RAIJ, B. van; CANTARELLA, H.; QUAGGIO, J.A; FERREIRA, M.E.; LOPEZ, A.S.; BATAGLIA, O.C. Análise química do solo para fins de fertilidade. Campinas: Fundação Cargill, 1987. 170p.

RAIJ, B. van; CANTARELLA, H.; QUAGGIO, J.A.;FURLANI, A.M.C. Recomendação de adubação e calagem para o Estado de São Paulo. 2.ed. Campinas: IAC, 1996. 285p. (Boletim Técnico, 100)

RICHTER, C.; HEILIGTAG, B.; NAGIEB, M.; MUKHALLALATI, $U$. Salinity effect in grown and ion concentration of eggfruit (Melongena solanum L.) and sweet pepper (Capssicum annuum L.). Tropenlandwirt, v.100, p.3-16, 1999.

SANTIAGO, C.L.; GOYAL, M.R. Nutrient uptake and solute movement in drip irrigated summer peppers. Journal of Agriculture of the University of Puerto Rico, v.69, p.63-68, 1985.

SILVA, C.A.; VALE, F.R. do; ANDERSON, S.J.; KOBAL, A.R. Mineralização de nitrogênio e enxofre em solos brasileiros sob influência da calagem e fósforo. Pesquisa Agropecuária Brasileira, v.34, p.1679-1689, 1999.

TADESSE, T.; NICHOLS, M.A.; FISHER, K.J. Nutrient conductivity effects on sweet pepper plants grown using a nutrient film technique: 1 . Yield and fruit quality. New Zealand Journal of Crop and Horticultural Science, v.7, p.229-237, 1999.

VALENZUELA, J.L.; ROMERO, L. Yield and optimum range nutrient in (Capsicum annuum L. cv. Lamuyo). Pythoninternational Journal of Experimental Botany, v.58, p.6375, 1996.

VASCONCELLOS, C.A.; FIGUEIREDO, A.P.H.D.; FRANÇA, G.E. de; COELHO, A.M.; BRESSAN, W. Manejo do solo e atividade microbiana em latossolo vermelho escuro da região de Sete Lagoas, MG. Pesquisa Agropecuária Brasileira, v.33, p.194208, 1998

WOSTEN, J.H.M.; GENUCHTEN, M. TH. van. Using texture and other soil properties to predict the unsaturated soil hydraulic functions. Soil Science Society of American Journal, v.52, p.1762-1770, 1988.

$\overline{\text { Recebido em } 15.03 .00}$ 